PoS $\quad \begin{aligned} & \text { PROCEEDINGS } \\ & \text { OF SCIENCE }\end{aligned}$

\title{
Physics Beyond The (Minimal Supersymmetric) Standard Model
}

\section{Thorsten Ohl*}

Institute for Theoretical Physics and Astrophysics, University of Würzburg

E-mail: ohlephysik.uni-wuerzburg.de

Third Linear Collider Physics School 2009 - LCPS2009

August 17 - 232009

Ambleside, $U K$

${ }^{*}$ Speaker. 


\section{Physics Beyond \\ The (Minimal Supersymmetric) Standard Model}

\section{Thorsten Ohl}

Institute for Theoretical Physics and Astrophysics, University of Würzburg

Linear Collider Physics School 2009

Ambleside, United Kingdom

August 17-24, 2009

\section{Contents}

Introduction

What have we seen?

What do most of us expect?

What do some of us hope for?

What might take us by surprise?

Perturbative All the Way Up To the Planck Scale

Supersymmetry

Extended Higgs Sectors

Perturbative Up To the Terascale (but not much further)

Extra Dimensions

Little Higgs / Gauge-Higgs Unification

Higgsless Models (a.k. a. (E)TC)

The Sgaugino $\Sigma^{0}$

LHC

Model Independent EFT Approach

VV $\rightarrow$ VV Below Threshold

Who Ordered That? 
- all collider experiments are compatible with a renormalization of some 18 parameters of the dim $\leqslant 4$ operators invariant under

$$
\mathrm{SU}(3)_{C} \times \mathrm{SU}(2)_{\mathrm{L}} \times \mathrm{U}(1)_{\mathrm{Y}}
$$

standard model

- the $\mathrm{SU}(2)_{\mathrm{L}} \times \mathrm{U}(1)_{Y}$ gauge symmetry appears to be spontaneously broken and vector bosons get their masses by eating a Goldstone boson, i. e. from a Higgs mechanism

- all current data are compatible with an elementary Higgs boson as the source of the Goldstone bosons

- if and only if interpreted as a fundamental renormalizable field theory, the data strongly favor a light Higgs boson

\section{What have we seen?}

- (cold) dark matter

$\because$ several independent observations (WMAP, rotation curves, gravitational lensing, structure formation, \&c.)

$\therefore$ very little wiggle room, any serious BSM model must leave room for CDM candidates

- (almost all) neutrinos have mass

- structurally not really BSM, b/c we can always add right handed singlets to obtain Dirac masses

$\because$ still: elegance of the seesaw mechanism(s) suggest Majorana masses w/associated higher mass scale $\approx 10^{10} \mathrm{TeV}$

$\therefore$ lepton flavor violation (e.g. $\mu^{ \pm} \rightarrow e^{ \pm} \gamma$ ) not unlikely

- dark energy

- solid evidence (WMAP, type IA supernova), but no hot particle physics candidates yet

- gravity

- the granddaddy of BSM physics

$\therefore$ there must be a new scale $m_{\text {Planck }} \gg v\left(\mathrm{w} / \mathrm{m}_{\text {Planck,4D }}=\mathcal{O}\left(10^{16}\right) \cdot v_{\mathrm{F}}\right)$ 
- Grand unification in some form is a central tenet of our discipline: "stuff becomes simpler at high energies - for a suitable notion of simple", e.g. $\mathrm{SU}(3)_{\mathrm{C}} \times \mathrm{SU}(2)_{\mathrm{L}} \times \mathrm{U}(1)_{Y} \rightarrow \mathrm{SU}(5)$

- requires BSM physics - some even at the Terascale

$\because$ assume gauge and Yukawa coupling unification

$\therefore$ there should be yet another new high scale

$\because$ does not work w/a desert above $v_{F}=254 \mathrm{GeV}$

$\therefore$ there should be yet another new threshold:

$m_{\text {Planck }} \gg m_{\text {threshold }}>v_{\mathrm{F}}$

- coexistence of widely separated scales raises naturalness concerns

$\because$ if there is a new much higher scale, we should explain the origin and stability of the lower scales

$\therefore$ new symmetries/particles for the protection of the EWSB scale

- caveat: viewed from the earth, the diameters of sun and moon appear to very finely tuned (anthropic principle: no astronomy, physics and higher mathematics w/o prediction of eclipses) ...

$\therefore$ most fertile ground for Terascale BSM models to date ...

- theoretical solution of the flavor problem

- number of generations

- mass and mixing hierarchies

- CP-violation

- observation of lepton flavor violation beyond $v$-mixing

- do we owe our existence to leptogenesis?

- is there a seesaw mechanism?

- are there Majorana masses? 
- Occam's razor might be dull: BSM physics can be a combination some or all of the above

- nature is often more messy than we hope

$\therefore$ be prepared!

- there are examples for "strange" stuff that doesn't fit nicely with (most of) our orthodoxy:

- Unparticles

- did anybody anticipate these propagators?

- Noncommutativity

- Lorentz invariance is hardwired in our brains ...

- nothing but the minimal SM plus an ad-hoc WIMP CDM candidate would be the biggest surprise of all

\section{Perturbative}

\section{All the Way Up To the Planck Scale}


- ironic: the ultimate new physics at LHC: the Higgs and nothing but the Higgs - the first fine-tuned theory that we once "understood"!

- NB: the fine tuning of the cosmological constant $\Lambda$ is worse, but nobody(?) claims to understand it ...

$\therefore$ current consensus: two options

- find a symmetry that protects the EWSB scale, and/or

- explain EWSB dynamically

- many scenarios allow to maintain our successful approach to new physics all the way up to the Planck scale:

- new physics in contact interactions $\propto 1 / \mathrm{m}_{\mathrm{NP}}^{2}$
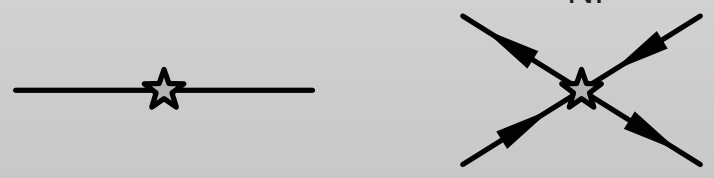

- can be interpreted as new particles of mass $\mathrm{m}_{\mathrm{NP}}$
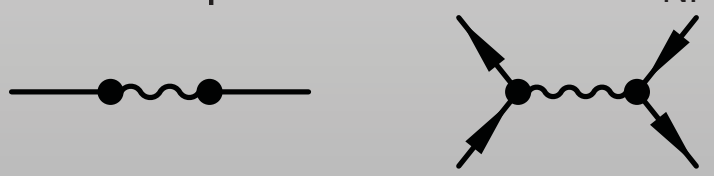

- examples: Z', see-saw mechanism

- for many (most?) SUSY is already contained in release 2.0 of the Standard Model which will be launched after a few $10 \mathrm{fb}^{-1}$ of LHC collisions have been analyzed in $201 x$

- very well motivated and well studied extension of the SM

- $\exists$ rich set of tools (dedicated and multi purpose) available - often very well tested in real applications

- $\exists$ multiple independent cross checked implementations of constrained versions of the MSSM, extensions in the works

$\because$ perturbatively renormalizable field theory allows clean factorization of tasks related to different scales, interfaces available, in particular SLHAn

- couplings from spectrum generators

- scattering amplitudes from diagrammatic tools

$\therefore$ all-in-one packages for LO event samples feasible (new model $\lesssim$ MA-thesis) 
- SUSY extensions of the SM require more than one Higgs doublet, but more general Higgs representations do not require SUSY

- just demand that

- $\rho \approx 1$ naturally

- FCNCs are naturally suppressed

- Glashow-Weinberg Criterion ['77] satisfied by 2HDM, w/mass eigenstates in reach of collider experiments

- popular source of CP-violation

- many phenomenological studies

- implemented in most (all?) all-in-one packages

\section{Perturbative \\ Up To the Terascale \\ (but not much further)}


- extra dimensions have been with us for a long, long time [Kaluza, Klein '21, '26] and string theory made them a necessity at the Planck scale

- Terascale extra dimensions became respectable in the late '90s [ADD '98, RS '99] (see also [Antoniadis '90])

- XDs play many (sometimes incompatible) rôles in Terascale particle physics

- real XD:

- can solve the hierarchy problem by a Terascale Planck mass

- introduce infinite Kaluza-Klein towers

- allow symmetry breaking by boundary conditions

- unitarize VV scattering by exchange of KK partners

- (ab)use the Xtra components of gauge fields as naturally light scalars

- metaphorical XD: symmetries in deconstructed dimensions

- holographical XD: powerful new description of strongly interacting models using the (conjectured!) AdS/CFT correspondence

- all degrees of freedom in XD represented by infinite Kaluza-Klein towers $m_{n}=n / R$ :

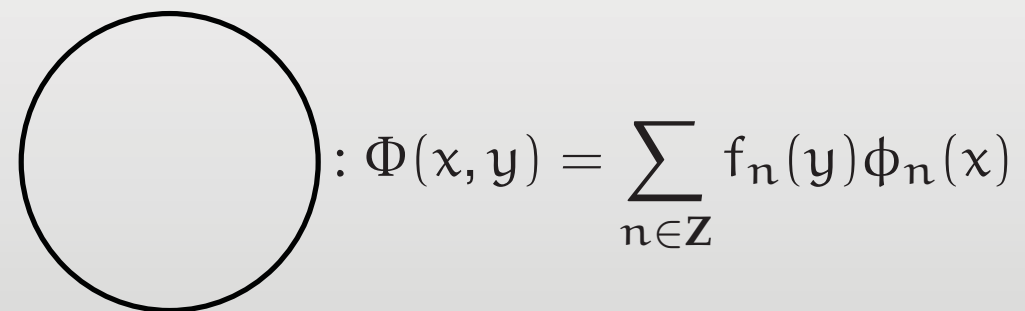

- orbifolding: identify points in the XD, e.g. $\Phi(x, y)=\Phi(x, 2 \pi-y)$

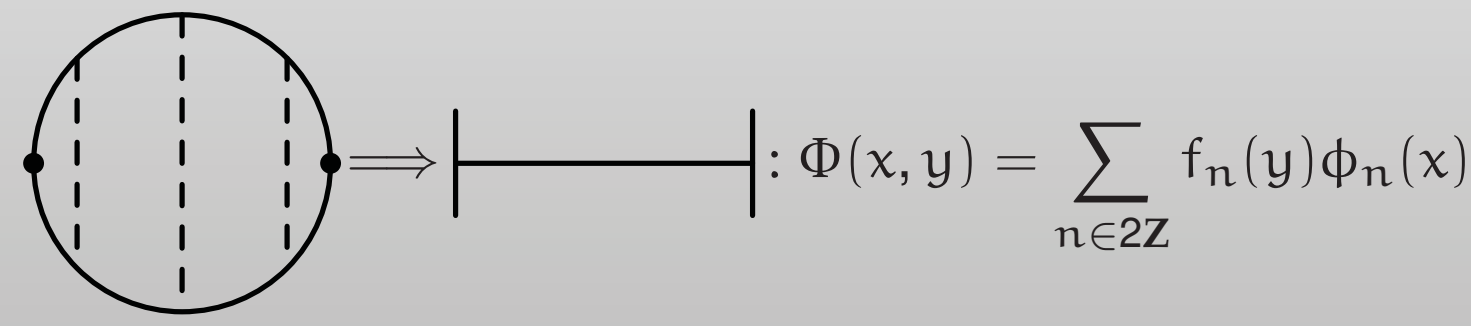

- "odd" modes are projected out

- fixed points (e.g. $y=0, \pi$ ) correspond to branes

$\therefore$ boundary conditions at the branes $\left(f_{n}(0)=0, \partial_{z} f_{n}(0)=0\right.$, etc. 
- replace continuous XD by discrete XD

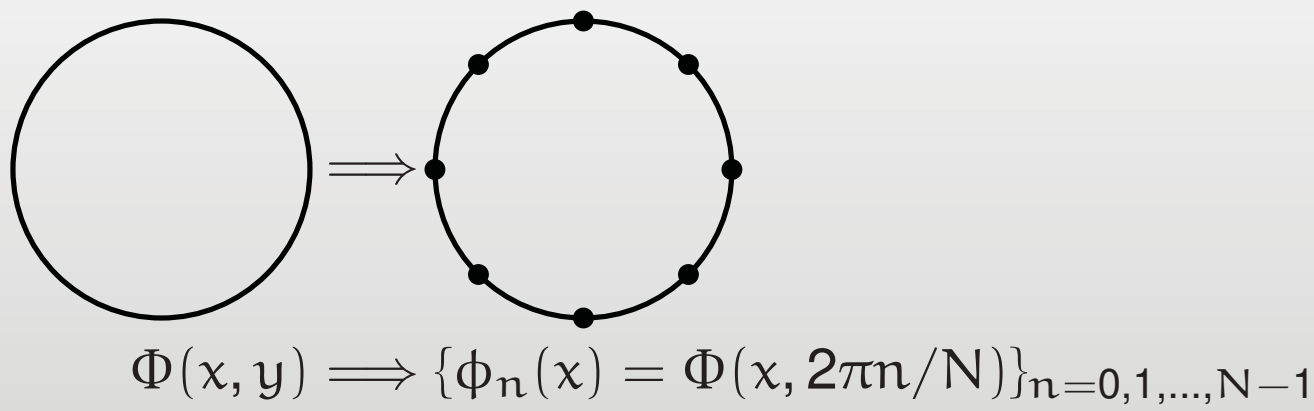

$\therefore$ finite dimensional representations of translation symmetry in $\mathrm{XD}$

- can be combined with orbifolding, of course

- 5D gauge theory is equivalent to a collection of 4D gauged nonlinear sigma models

$$
\int d y \frac{1}{4} \operatorname{tr}\left(F_{\mu \nu} F^{\mu \nu}\right) \Rightarrow \text { "lattice" } \Rightarrow \frac{1}{4} \sum_{n=1}^{N} \operatorname{tr}\left(D_{\mu} \Phi^{\dagger}\left(x, y_{n}\right) D^{\mu} \Phi\left(x, y_{n}\right)\right)
$$

- NB: one loop quadratic divergencies for uneaten goldstone bosons cancel from remnant of translational symmetry!

\section{Holographic XD}

- replace flat XD by warped XD:

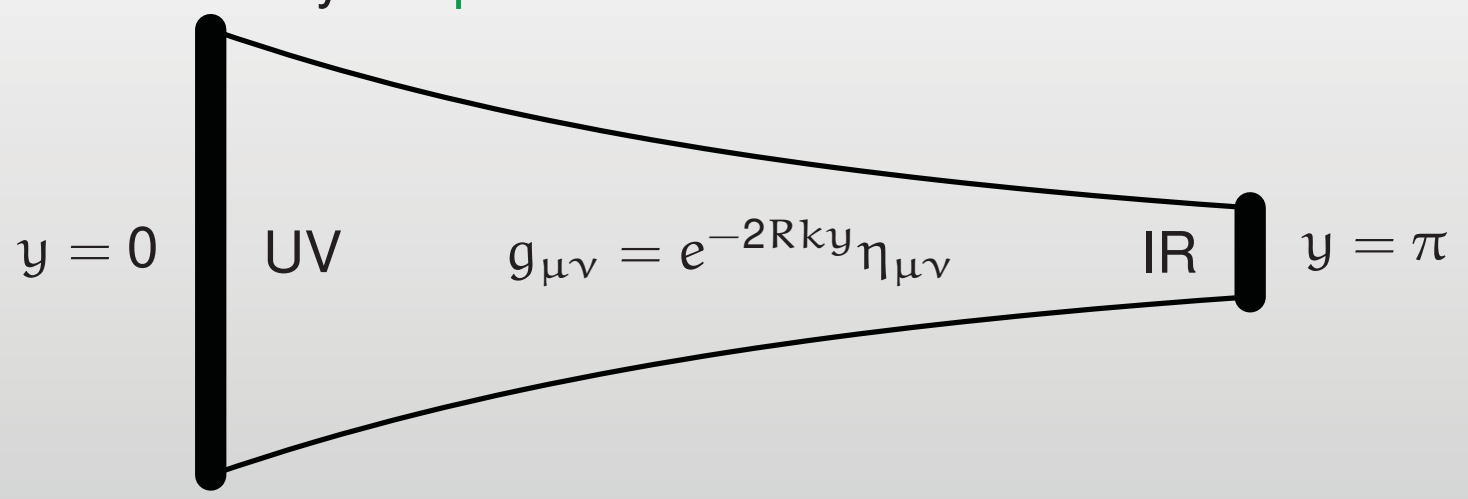

- NB: warped XD play a dual rôle

- warp factor creates hierarchy $m / M \approx e^{-R k \pi}$

- if the AdS/CFT correspondence is correct, we can describe a strongly coupled 4D theory by a dual weakly coupled 5D theory!

$\therefore$ the technicolor and composite Higgs models of the '70s and ' 80 s have been resurrected as models on AdS5!

- can be combined with deconstruction, of course 
- Little Higgs started life as deconstructed XD

$$
\begin{aligned}
& \sum_{n} \bigcap^{n} \propto \Lambda^{2} . \underbrace{\sum_{-N / 2<n \leqslant N / 2} \cos \left(2 \pi \frac{n}{N}+\phi\right)+g \ln \Lambda}_{-0} \\
& =0
\end{aligned}
$$

- can be reproduced by internal symmetry breaking pattern, e. g.

$$
\mathrm{SU}(5) \rightarrow \mathrm{SO}(5)
$$

- drawbacks:

- hierarchy propblem merely postponed

- two loop contributions remain quadratically divergent:

$$
\Lambda: 1 \mathrm{TeV} \rightarrow 10 \mathrm{TeV}
$$

- Randall-Sundrum started with only gravity in the bulk (motivation: open string endpoints confined to D-branes)

- warp factor softens hierarchy from power to logarithm

- also: smallness of $\rho-1$ (\& other EW precision observables) not natural in EWSB by boundary conditions in flat XD

- can be explained by similar exponential suppression of the symmetry breaking sector [Csaki et al. '03]

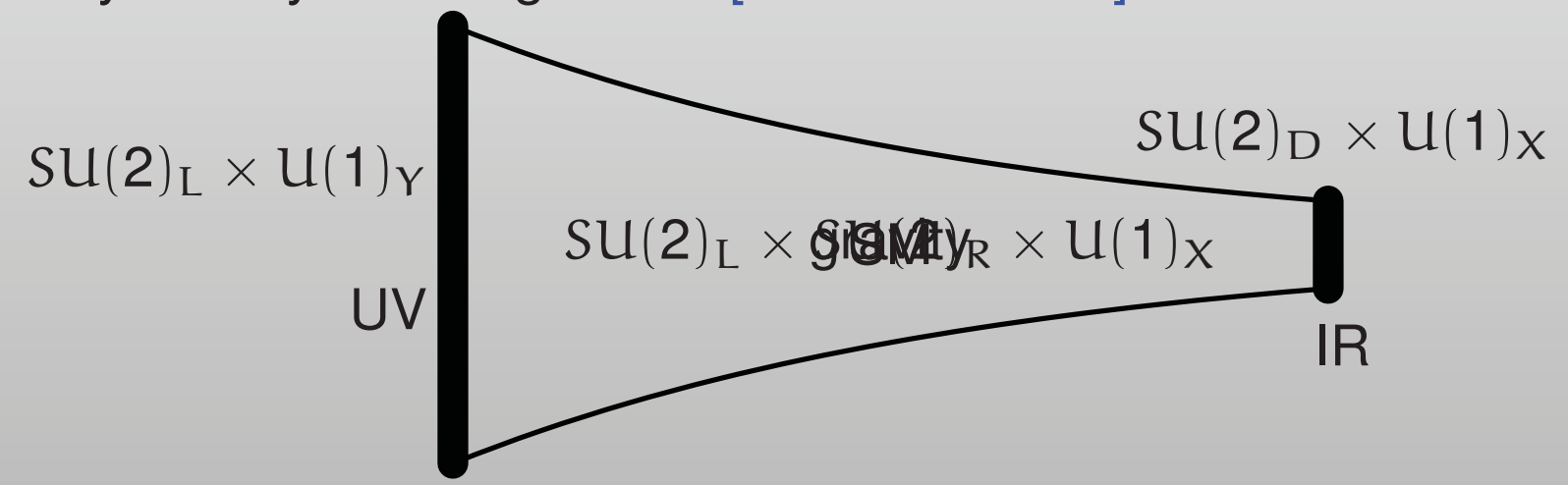

- couplings from overlap integrals in the extra dimension 
- even higgsless models must provide reasonable CDM candidates

$\because$ warp factors destroy Kaluza-Klein parity

$\therefore \nexists$ stable Lightest KK Particle (LKP)

$\therefore$ some additional physics $B S M$ required

- two branes with parity [Agashe et al. '07, Panico et al. '08]

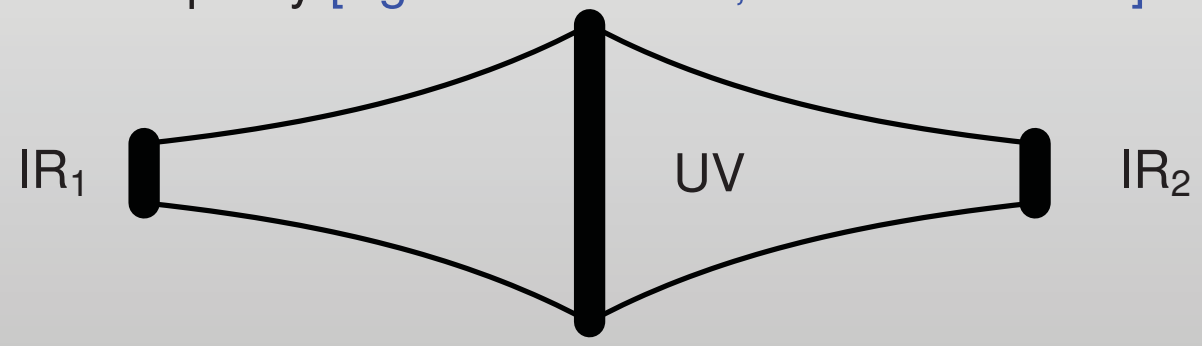

- R-parity conserving SUSY in warped 5D [Knochel, TO '08]

$\because$ SUSY well motivated to appear in UV completions of any effective model, including higgsless models

\section{XD w/SUSY}

- flat 5D $N=1$ can be mapped to 4D $N=2$

- $N=2$ SUSY broken by warp factor, only one y-dependent global $\mathrm{N}=1$ SUSY compatible with the metric ("killing spinors")

$$
\xi(y)=e^{-R k y / 2}\left(\begin{array}{c}
\xi_{\alpha}^{0} \\
0
\end{array}\right)
$$

$\therefore$ remaining degeneracy must be lifted by soft breaking

- most elegantly by boundary conditions on the UV-brane

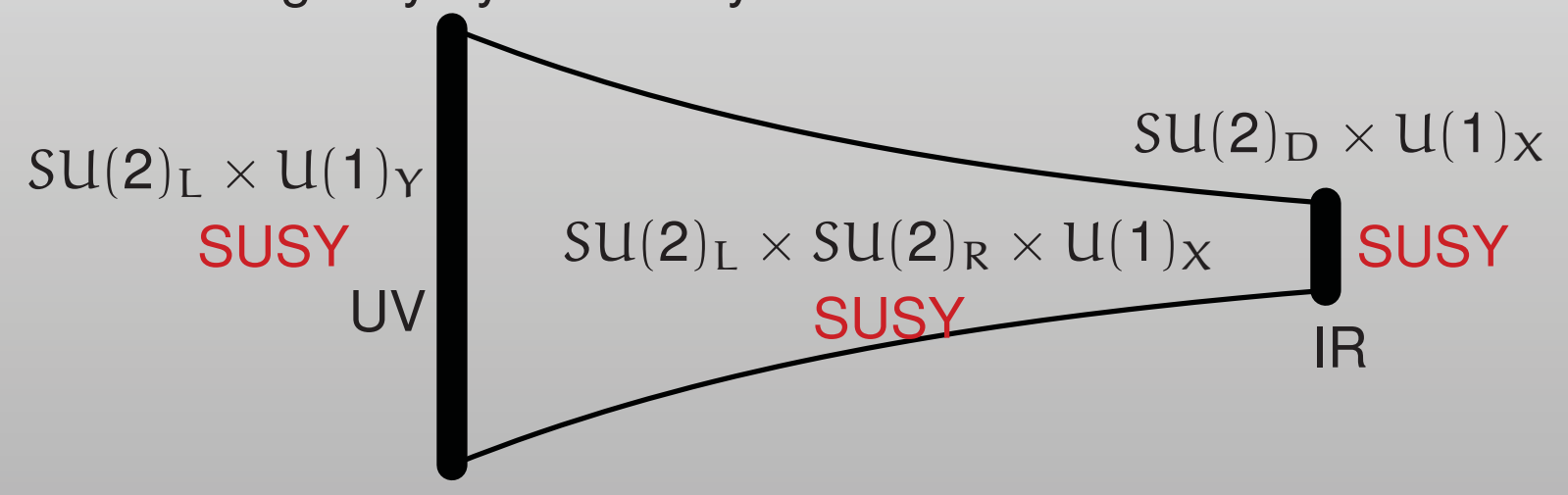


- spectrum of gauge bosons and matter together with $\mathrm{KK}$ and SUSY partners:

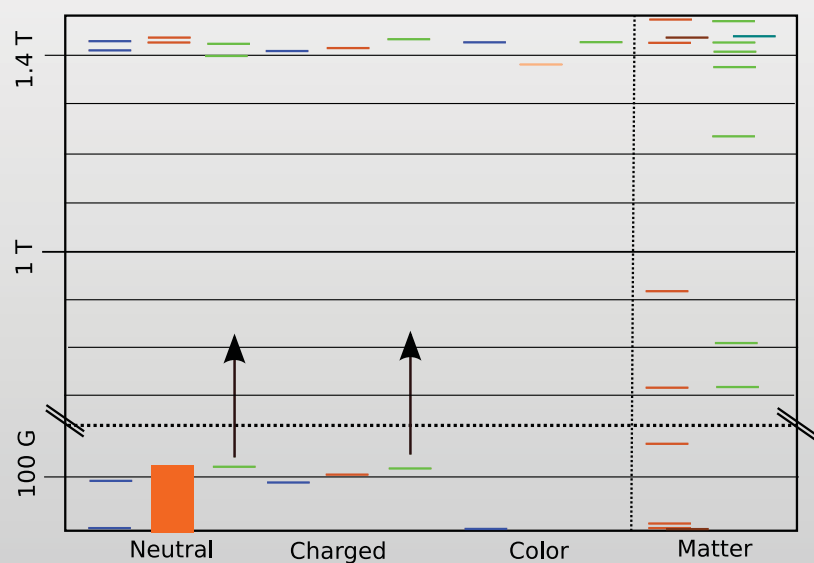

- couplings of the heavy gauge scalars ("sgauginos"),
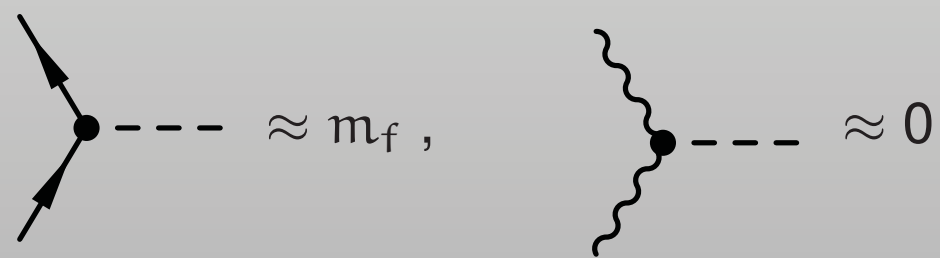

$\therefore$ Higgs-like w/o vector boson fusion

- estimate neutralino relic density freezeout at $\approx \mathrm{m}_{\chi} / 20$

$$
1 / \Omega h^{2} \propto\langle\nu \sigma(\chi \chi \rightarrow W W)\rangle+\underbrace{\langle v \sigma(\chi \chi \rightarrow f f)\rangle}_{\approx 0 \text { for } m_{\chi}<m_{t}, m_{\chi} \ll m_{\tilde{f}}}
$$

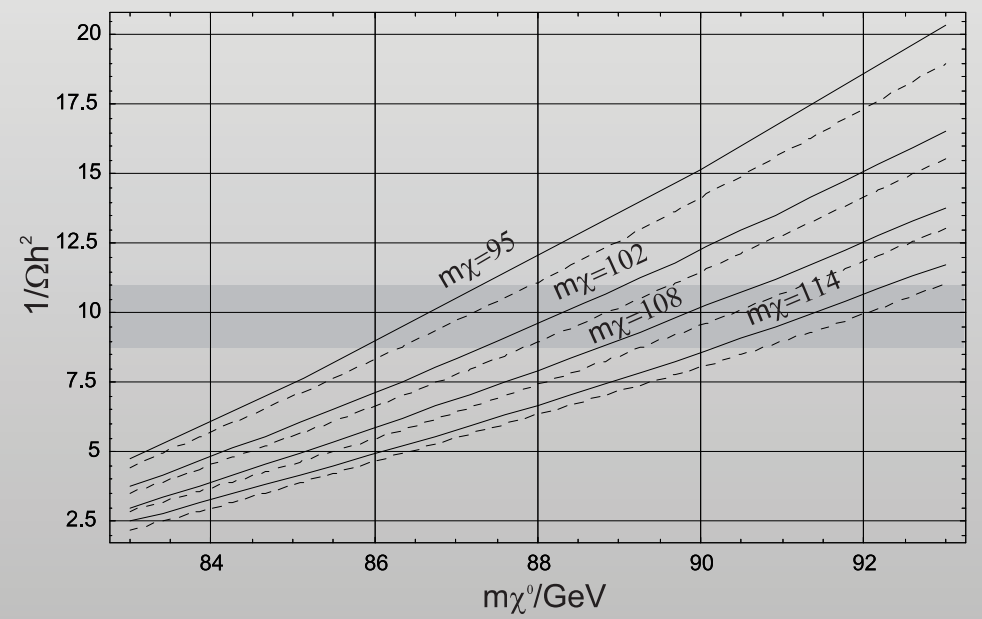

- Very good agreement with current WMAP data possible 
- scalar $\Sigma$ as $\mathcal{N}=2$ partner of the gauge bosons

- no $\Sigma A_{\mu} A_{\mu}$ interaction $\Longrightarrow$ no Vector boson fusion

$\because$ Interaction with fermions $\mathcal{L}_{\Sigma f \bar{f}}=g \frac{1}{(k z)^{5}} \times\left(\Sigma_{0}+i A_{5}\right) \eta+$ h. c.

$y_{\text {eff }}^{0} \propto\left\langle\Sigma_{0} \eta_{L} \chi_{L}\right\rangle+\left\langle\Sigma_{0} \eta_{R} \chi_{R}\right\rangle \begin{cases}m_{f}=0: & \eta_{R}=\chi_{L}=0 \\ m_{f}>0: & \text { contributions from } \eta_{R}, \chi_{L}\end{cases}$

$\therefore y_{\text {eff }}^{0}$ grows with fermion mass, similar to SM Higgs: $y_{\text {eff }}^{0} \approx \frac{y_{H}}{3}$

$\therefore \Sigma_{0}$ production similar to SM
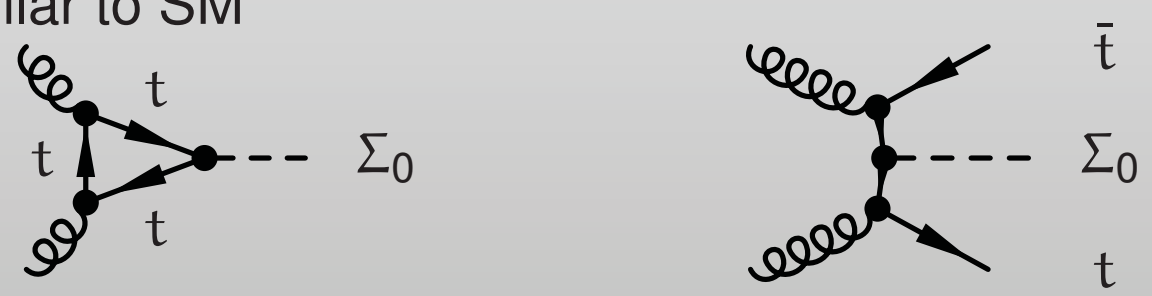

$\because$ However: the sfermion partners of SM fermions are projected out by boundary conditions

$\therefore$ (potentially) large mass corrections

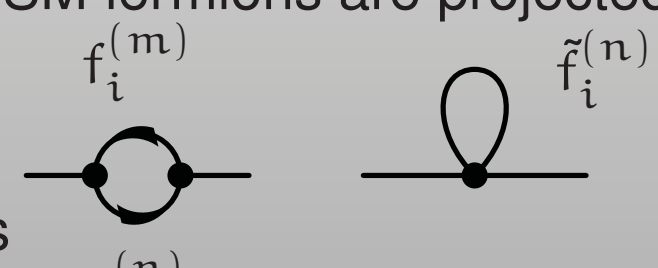
$f(n)$

\section{LHC}

- tree level contributions to associated heavy quark and LSP pair production with a $q \bar{q}$ initial state.
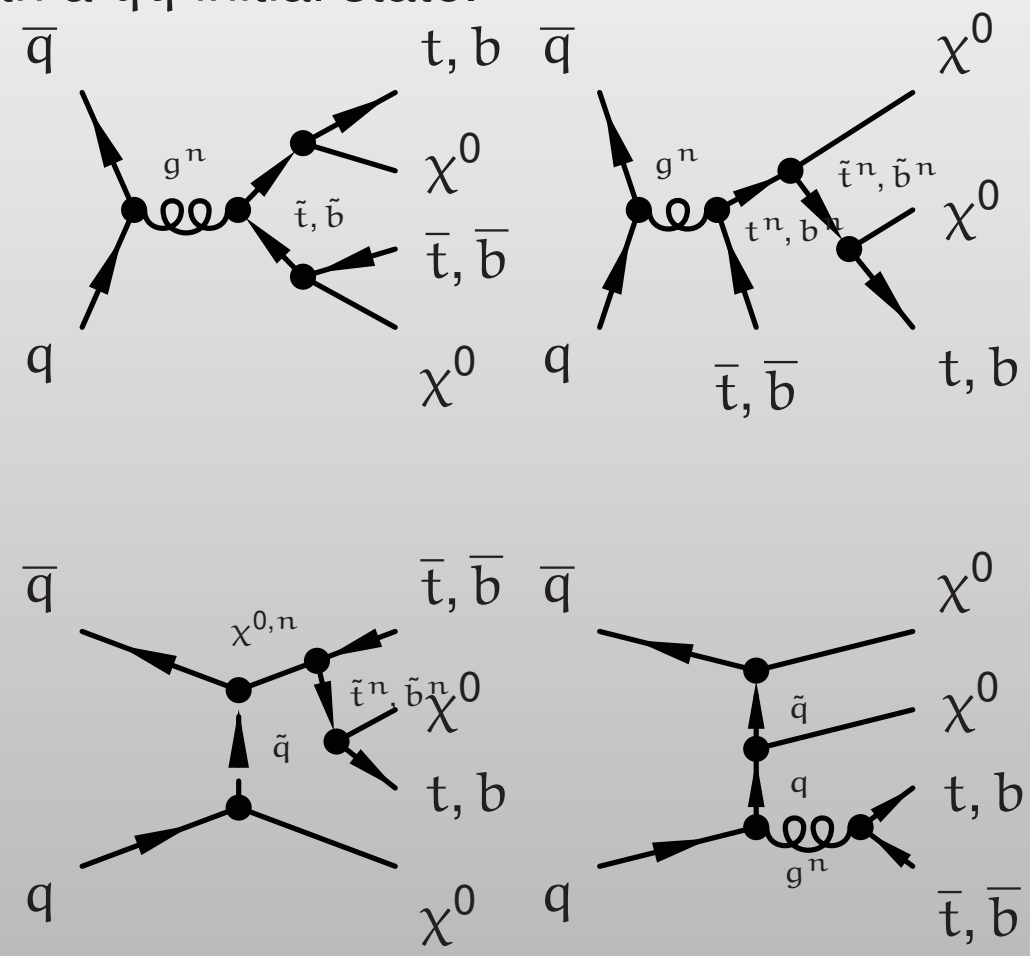
- tree level contributions to associated heavy quark and LSP pair production with a gg initial state.

$\mathrm{g}$

$t, b \quad g$

9
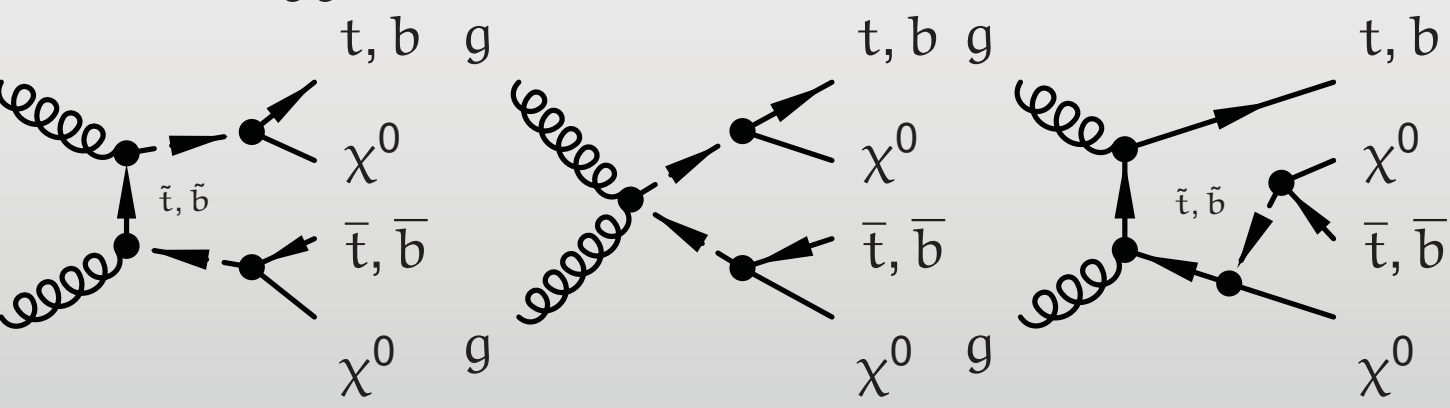

9

$t, b \quad g$
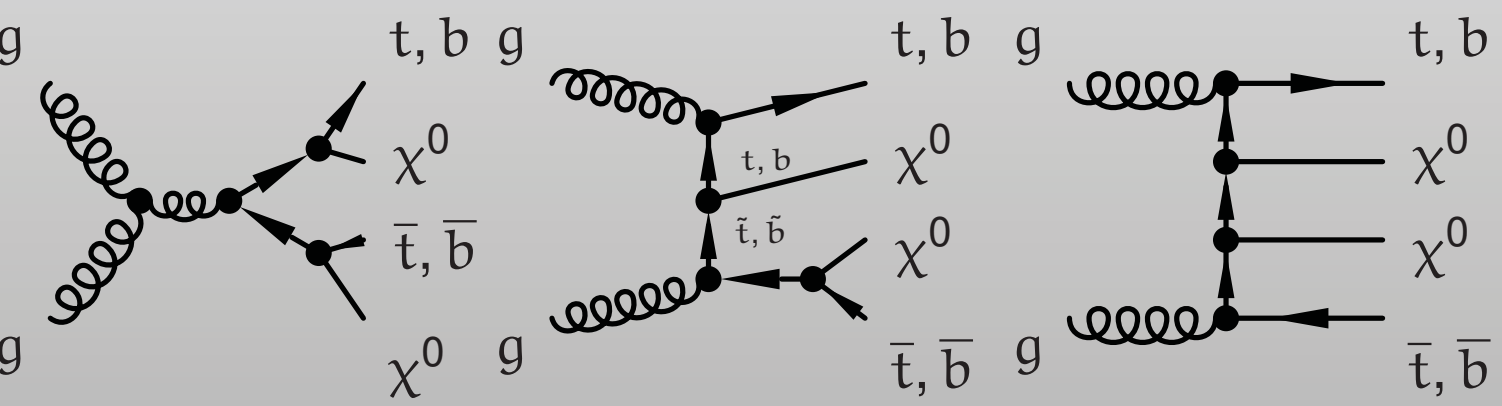

- Model implemented by in WHIZARD [Kilian/TO/Reuter]

- commercial break:

- fully automated Monte Carlo event generator generator (emphasis on BSM physics, w/and w/o SUSY)

http://whizard.event-generator .org (or hepforge.org)

- $\alpha$-Version of Version 2 recently completed (still working out Fortran 2003 compiler kinks)

- hadron colliders no longer an afterthought (Version 1 sometimes revealed its TESLA/ILC origins)

- Kinematic cuts

\begin{tabular}{c|c|c|c|c} 
Variable & $\mathrm{I}$ & $\mathrm{Il} .1$ & $\mathrm{Il} .2$ & II.3 \\
\hline \hline $\mathrm{P}_{\mathrm{T}}(\mathrm{q}), \mathrm{P}_{\mathrm{T}}(\overline{\mathrm{q}})$ & - & $>100 \mathrm{GeV}$ & $>300 \mathrm{GeV}$ & $>100 \mathrm{GeV}$ \\
\hline$\Delta \phi(\mathrm{q}, \overline{\mathrm{q}})$ & - & - & - & {$\left[0,140^{\circ}\right]$}
\end{tabular}

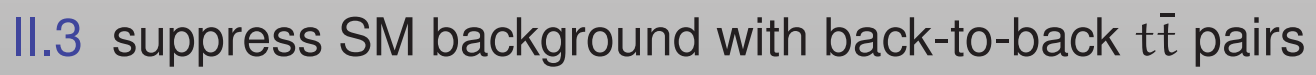


- Missing energy in neutralino LSP pair production in association with top pairs (SM background: $v \bar{v} t \bar{t})$ ):
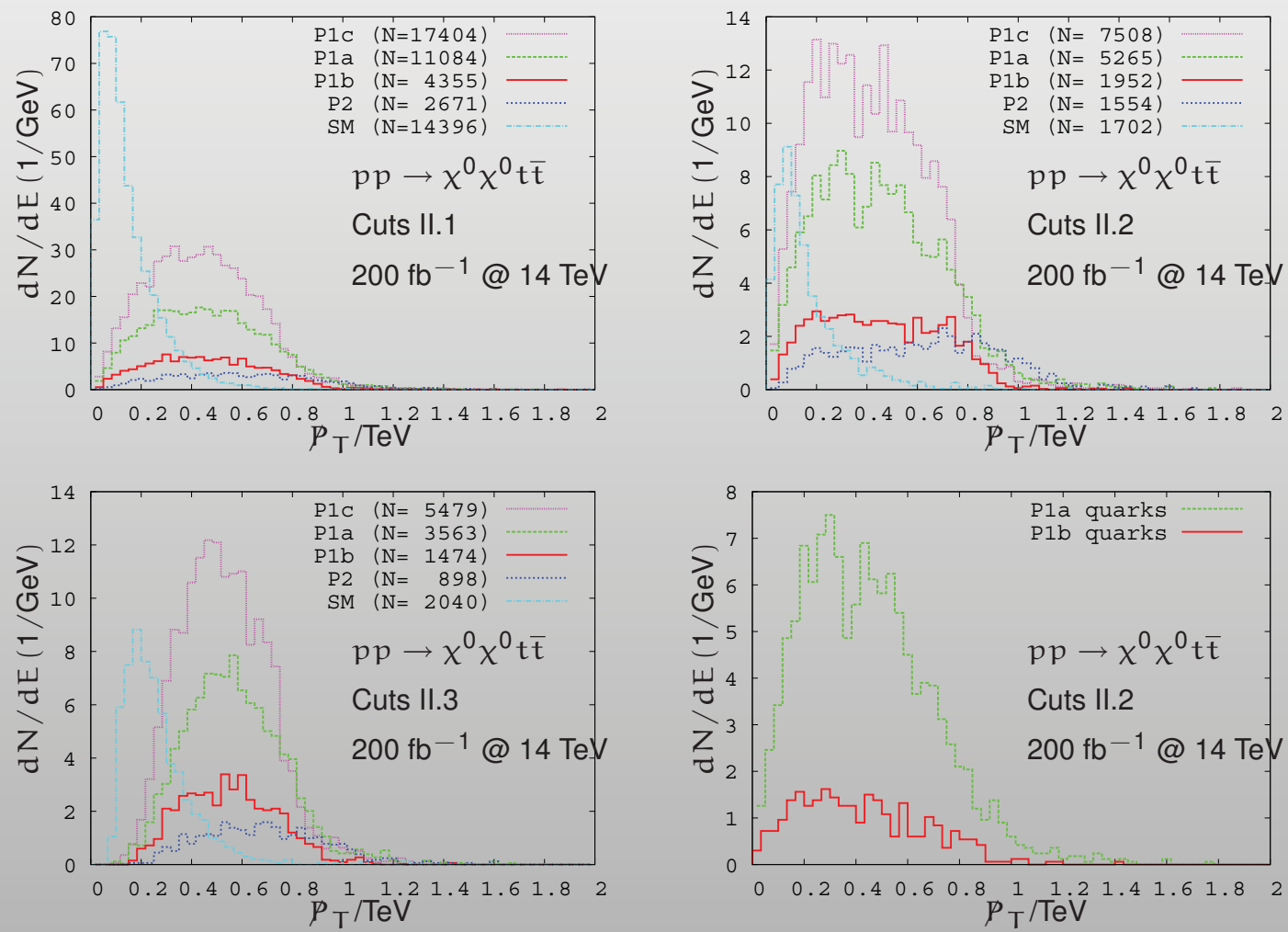

\section{Model Independent EFT Approach}


- Most conservative approach:

- use only observed degrees of freedom

- implement observed exact and broken symmetries

$\therefore$ effective chiral Lagrangian for $\mathrm{SU}_{\mathrm{L}}(2) \times \mathrm{SU}_{\mathrm{R}}(2) \rightarrow \mathrm{SU}_{\mathrm{C}}(2)$ breaking

$$
\mathcal{L}=\frac{1}{4} \operatorname{tr}\left(\left[D_{\mu}, D_{v}\right]\left[D^{\mu}, D^{v}\right]\right)+\frac{v_{F}^{2}}{2} \operatorname{tr}\left(D_{\mu} U^{\mu} U\right)+\ldots
$$

- dependence of $\mathrm{V} V \rightarrow \mathrm{V} V$ and $\mathrm{V} V \rightarrow \mathrm{t} \overline{\mathrm{t}}$ scattering on dim-4 operators
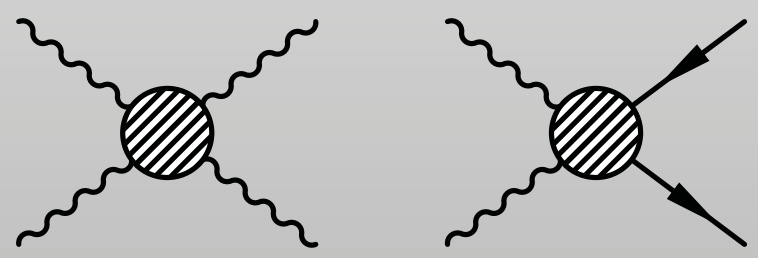

studied for ILC ( $\left.e^{+} e^{-} \rightarrow 6 f / 8 f\right)$ and LHC $(p p \rightarrow 6 f / 8 f)$

- custodial SU(2) ${ }_{c}$ conserving:

$$
\begin{aligned}
& \mathcal{L}_{4}=\alpha_{4} \operatorname{tr}\left[\mathrm{V}_{\mu} \mathrm{V}_{\nu}\right] \operatorname{tr}\left[\mathrm{V}^{\mu} \mathrm{V}^{\nu}\right] \\
& \mathcal{L}_{5}=\alpha_{5} \operatorname{tr}\left[\mathrm{V}_{\mu} \mathrm{V}^{\mu}\right] \operatorname{tr}\left[\mathrm{V}_{\nu} \mathrm{V}^{\nu}\right]
\end{aligned}
$$

where $\mathrm{V}_{\mu}=\mathrm{U}^{\dagger} \mathrm{D}_{\mu} \mathrm{U}$

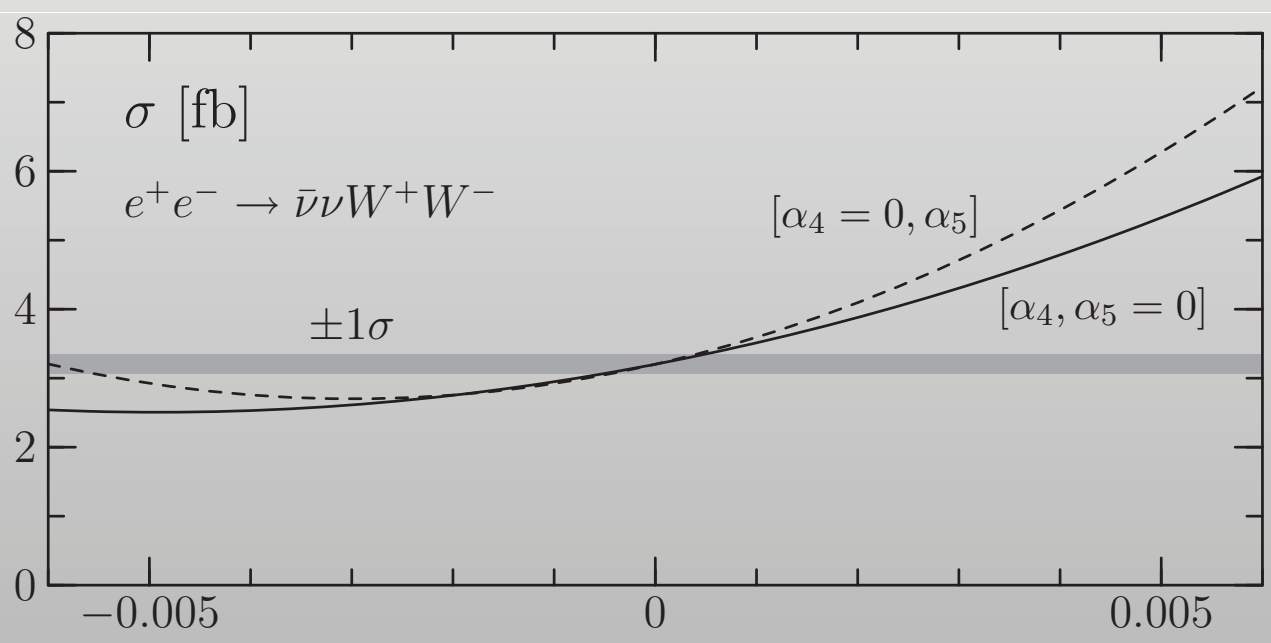


- our 1997 Dream Machine could probe $\alpha_{4,5}$ at the "magic" $\mathcal{O}\left(10^{-3}\right) \lesssim 1 /\left(16 \pi^{2}\right)$ suggested by naive dimensional analysis

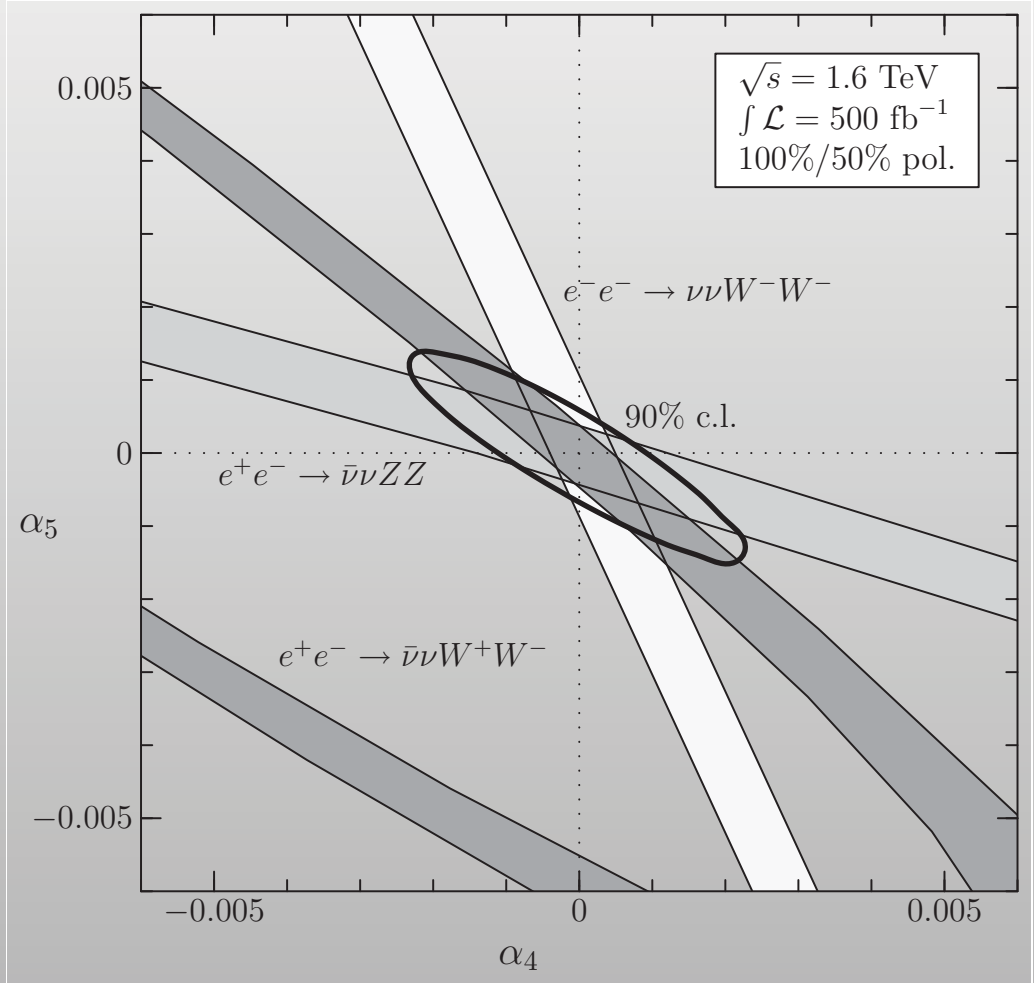

\section{EFT VV $\rightarrow$ VV Below Threshold}

- custodial SU(2) $)_{\mathrm{c}}$ violation:

$$
\begin{aligned}
& \mathcal{L}_{6}=\alpha_{6} \operatorname{tr}\left[\mathrm{V}_{\mu} \mathrm{V}_{\nu}\right] \operatorname{tr}\left[\mathcal{T} \mathrm{V}^{\mu}\right] \operatorname{tr}\left[\mathcal{T} \mathrm{V}^{\nu}\right] \\
& \mathcal{L}_{7}=\alpha_{7} \operatorname{tr}\left[\mathrm{V}_{\mu} \mathrm{V}^{\mu}\right] \operatorname{tr}\left[\mathcal{T} \mathrm{V}_{\nu}\right] \operatorname{tr}\left[\mathcal{T} \mathrm{V}^{\mathcal{\nu}}\right]
\end{aligned}
$$

where $\mathcal{T}=U \tau_{3} U^{\dagger}$.

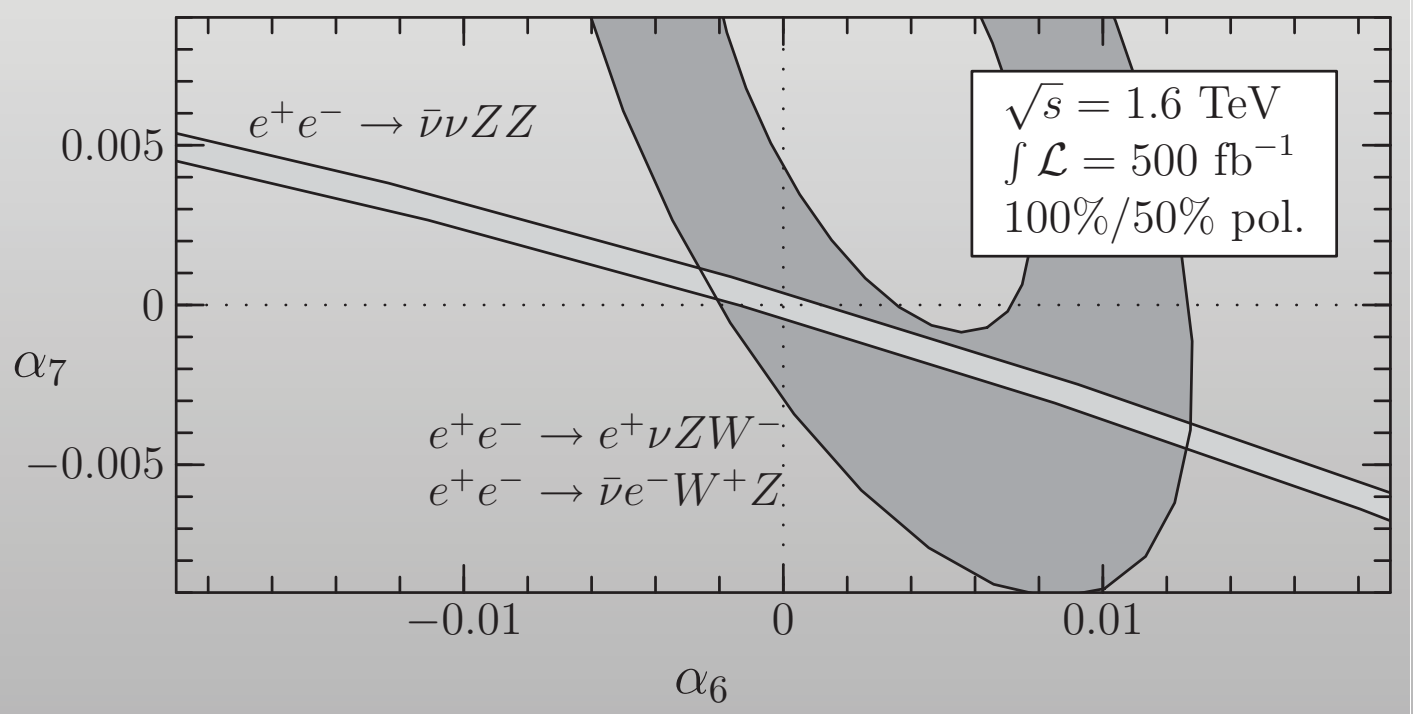




\section{Who Ordered That?}

- Quantum mechanics: measurements of coordinate and momentum are complementary

$$
\Delta \mathrm{x}_{\mathrm{i}} \cdot \Delta \mathrm{p}_{\mathrm{j}} \geqslant \hbar / 2 \cdot \delta_{i j}
$$

More formal: the corresponding operators don't commute

$$
\left[x_{i}, p_{j}\right]=x_{i} p_{j}-p_{j} x_{i}=i \hbar \delta_{i j}
$$

- Currently no exp. evidence for complementary coordinate pairs:

$$
\Delta x_{\mu} \cdot \Delta x_{v} \stackrel{?}{=} 0 \quad \Leftrightarrow \quad\left[x_{\mu}, x_{v}\right] \stackrel{?}{=} 0
$$

- nevertheless

$$
\left[\hat{x}_{\mu}, \hat{x}_{v}\right]=i \theta_{\mu \nu}=i \frac{C_{\mu \nu}}{\Lambda_{N C^{2}}}
$$

possible, as long as characteristic energy scale $\wedge_{\mathrm{NC}}$ large and corresponding minimal area in the $e_{\mu} \wedge e_{\nu}$-plane

$$
a_{N C}=l_{N C}^{2}=1 / \Lambda_{N C}^{2}
$$

small compared to the resolution of present experiments. 
Why is it interesting?

- Fundamental length scale

- $x_{\mu}$-continuum $\Rightarrow$ lattice of eigenvalues of operators $\hat{x}_{\mu}$ (lattice constant $\sim 1 / \wedge_{\mathrm{NC}}$ ) [Snyder, Wess]

- smooth cut off of some divergent contributions $E>\Lambda_{N C}$ in quantum gravity (cf. $\hbar$ and black body radiation)

$\because$ internal and space-time symmetries do not commute any more

$\therefore$ richer symmetry structure

- String theory

- NCQFT is low energy limit of certain string theories [Seiberg, Witten]

- more than 2000 citations for a single paper written in 1999 ...

- no prediction for the value of $\Lambda_{\mathrm{NC}}$

- special (simplest) case: $\theta^{\mu \nu}$ constant $4 \times 4$-matrix:

$$
\left[\hat{x}^{\mu}, \hat{x}^{\nu}\right]=i \theta^{\mu \nu}=i \frac{1}{\Lambda_{N C}^{2}} C^{\mu \nu}=i \frac{1}{\Lambda_{N C}^{2}}\left(\begin{array}{cccc}
0 & -E^{1} & -E^{2} & -E^{3} \\
E^{1} & 0 & -B^{3} & B^{2} \\
E^{2} & B^{3} & 0 & -B^{1} \\
E^{3} & -B^{2} & B^{1} & 0
\end{array}\right)
$$

\section{Noncommutative Space Time}

- simpler, but equivalent realization: replace all point products of functions of noncommuting coordinates

$$
(f \cdot g)(\hat{x})=f(\hat{x}) g(\hat{x})
$$

by Moyal-Weyl-*-products of functions of commuting coordinates:

$$
(f * g)(x)=f(x) e^{\frac{i}{2} \overleftarrow{\partial^{\mu}} \theta_{\mu \nu}} \overrightarrow{\partial^{v}} g(x)=f(x) g(x)+\frac{i}{2} \theta_{\mu \nu} \frac{\partial f(x)}{\partial x_{\mu}} \frac{\partial g(x)}{\partial x_{\nu}}+\mathcal{O}\left(\theta^{2}\right)
$$

- then $\left(x_{\mu} * x_{v}\right)(x)=x_{\mu} x_{v}+\frac{i}{2} \theta_{\mu \nu}$ and in particular

$$
\left[x_{\mu}, x_{v}\right](x)=\left(x_{\mu} * x_{v}\right)(x)-\left(x_{v} * x_{\mu}\right)(x)=i \theta_{\mu \nu}
$$

- new interaction vertices among gauge and matter fields from expanding Moyal-Weyl-*-products and Seiberg-Witten-Maps as determined by gauge invariance

$$
g(\overline{\hat{\psi}} * \hat{\mathcal{A}} * \hat{\psi})(x)=g \bar{\psi}(x) \not \mathcal{X}(x) \psi(x)+\mathcal{O}(\theta)
$$


- e.g. at $O(\theta)$ with all momenta outgoing

$$
\begin{aligned}
& \sim=\mathrm{ig} \cdot \frac{\mathrm{i}}{2}\left[(k \theta)_{\mu} \not p+(\theta p)_{\mu} k-(k \theta p) \gamma_{\mu}\right] \\
& =i g^{2} \cdot \frac{i}{2}\left[\begin{array}{r}
\left(\theta\left(k_{1}-k_{2}\right)\right)_{\mu_{1}} \gamma_{\mu_{2}}-\left(\theta\left(k_{1}-k_{2}\right)\right)_{\mu_{2}} \gamma_{\mu_{1}} \\
-\theta_{\mu_{1} \mu_{2}}\left(k_{1}-k_{2}\right)
\end{array}\right]
\end{aligned}
$$

- canonical NC extension of the SM known to $\mathcal{O}\left(\theta^{2}\right)$

\section{Noncommutative Space Time}

standard acceptance cuts and $85 \mathrm{GeV}<\mathrm{m}_{\ell^{+} \ell^{-}}<97 \mathrm{GeV}$, $200 \mathrm{GeV}<\mathrm{m}_{\ell^{+} \ell^{-} \gamma}<1 \mathrm{TeV}, 0<\cos \theta_{\gamma}^{*}<0.9$, $\cos \theta_{Z}>0$ and $\cos \theta_{\gamma}>0$ (favoring $\bar{q} q$ over $\left.q \bar{q} !\right)$

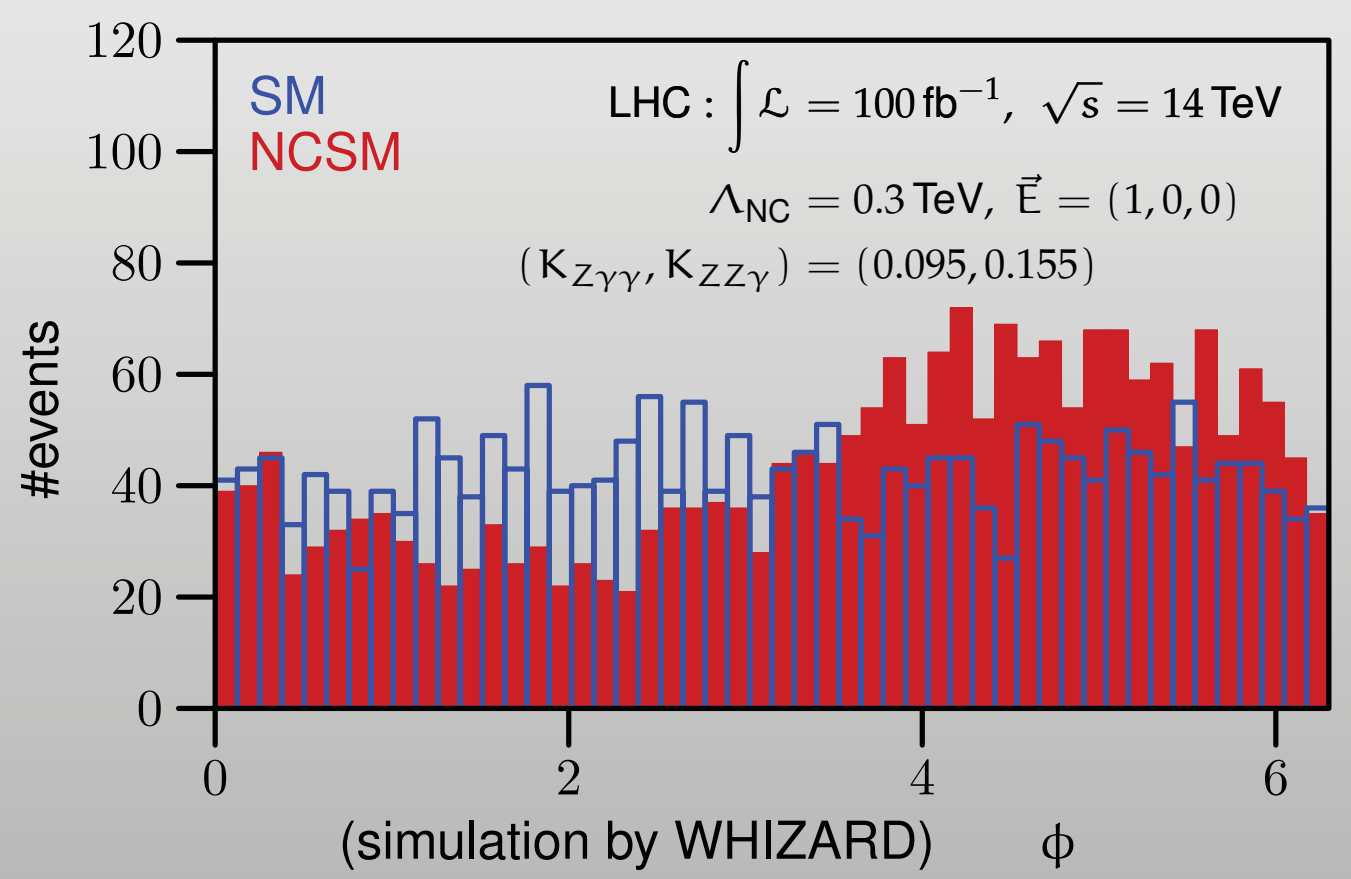


likelihood fits for $\Lambda_{\mathrm{NC}}=500 \mathrm{GeV}$ [Alboteanu, T. O., Rückl, PRD74]
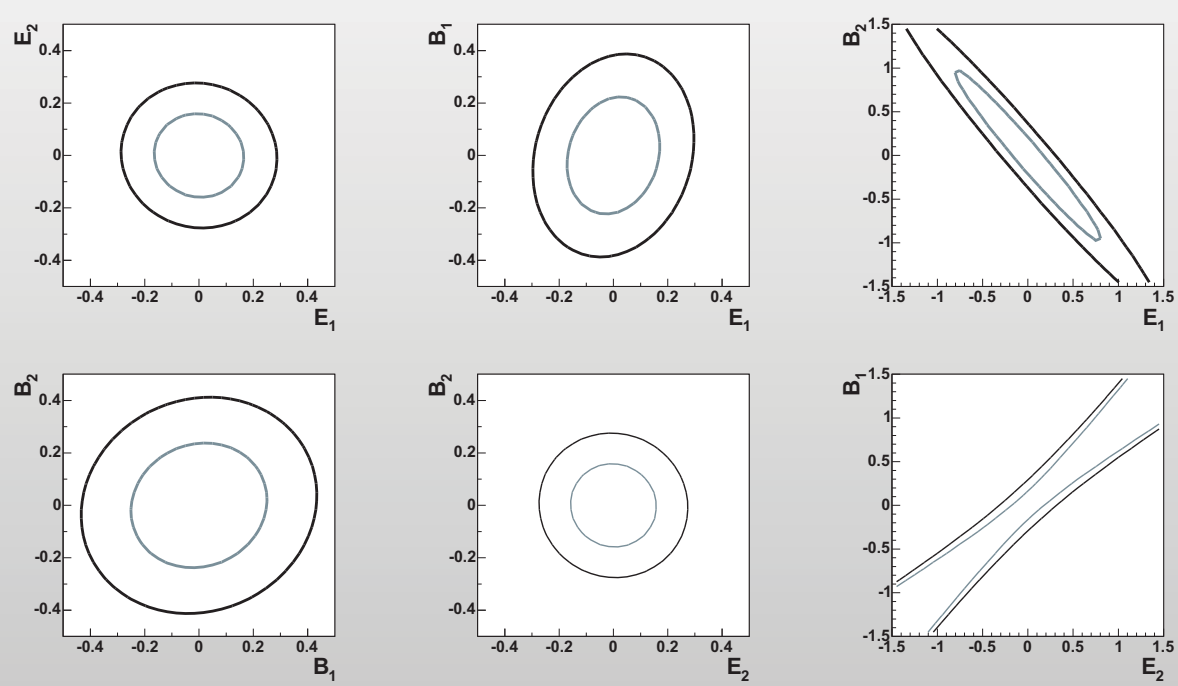

- only the expected kinematical correlations of $\left(E_{1}, B_{2}\right)$ and $\left(E_{2}, B_{1}\right)$

$\therefore \Lambda_{\mathrm{NC}} \gtrsim 1 \mathrm{TeV}$ can be easily probed at the LHC

- unfortunately, hard to reconcile with Lorentz violation bounds from atomic physics and astronomy

\section{Facts of Life ...}

$\because$ Light fermions couple very weakly to the Electroweak Symmetry Breaking sector

- Standard Model Yukawa Couplings

$$
\mathcal{L} \supset \frac{m_{f}}{\nu_{F}} H \bar{\psi}_{f} \psi_{f} \Longrightarrow \frac{d \sigma}{d \Phi}(f \bar{f} \rightarrow H) \propto \frac{m_{f}^{2}}{v_{F}^{2}}
$$

- generically in any chiral Effective Field Theory description

$$
\mathcal{L} \supset m_{f} \bar{\psi}_{f} \exp \left(i \frac{\Phi}{v_{F}}\right) \psi_{f} \Longrightarrow \frac{d \sigma}{d \Omega}(f \bar{f} \rightarrow \Phi) \propto \frac{m_{f}^{2}}{v_{F}^{2}}
$$

$\therefore$ cross sections for the direct excitation of the EWSB sector at LHC $(u, d)$ and ILC $\left(e^{ \pm}\right)$are strongly suppressed 
$\because$ couplings of all fermions except top have been measured very precisely at LEP 1

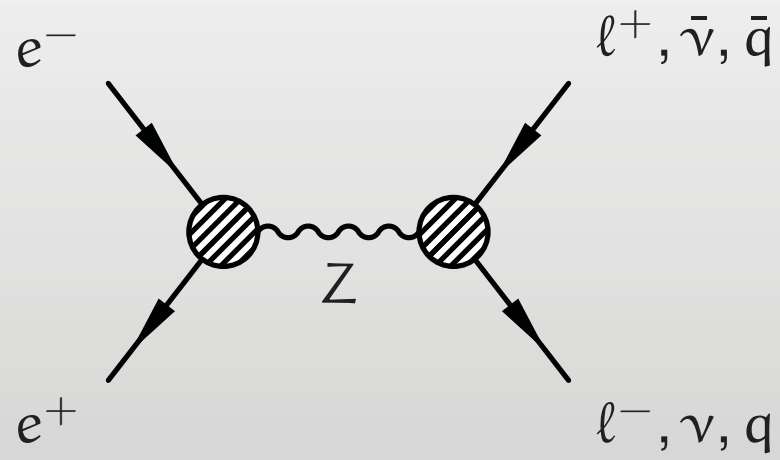

$\because$ all observed Flavor Changing Neutral Currents can be explained by penguin and box diagrams

$\therefore$ new particles in models of any new physics are very likely to be fermiophobic (with an exception for top quarks)

(210) direct production cross sections for new gauge bosons \&c. at ILC and LHC strongly suppressed again!

$\therefore$ new physics appears to suffer from fermiophobia!

$\therefore$ produce excitations of the EWSB sector (e.g. Higgs bosons) in association with heavy particles (i. e. $\mathrm{m}=\mathcal{O}\left(v_{\mathrm{F}}\right)$ ) via their known gauge couplings

- e.g. top-quarks

- or $W$ and $Z$ bosons

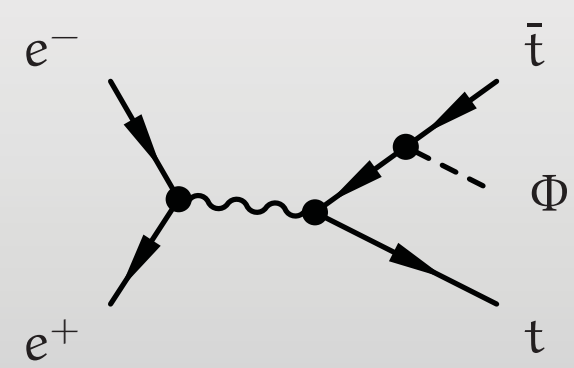

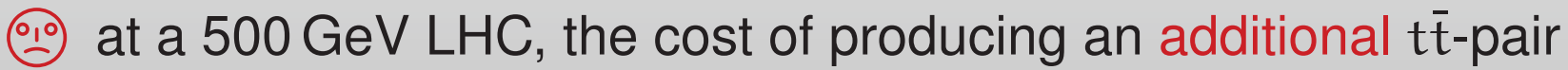
pushes it back to the threshold of a light Higgs:

$$
500 \mathrm{GeV}-2 m_{t}=150 \mathrm{GeV}
$$

- LHC advantage: colored new physics must have a large cross section in gluon-gluon scattering

- NB: guaranteed by universality of the strong coupling 
- Trick: generate almost real massive gauge bosons ( $\mathrm{W}^{ \pm}$and $\mathrm{Z}$ ) with known gauge couplings by bremsstrahlung off light fermions and let them scatter

- cross section suppressed by additional gauge couplings

$$
\left(\frac{\alpha}{\pi}\right)^{2} \approx 5 \cdot 10^{-6}
$$

- but enhanced by

$$
\left(\frac{m_{W, z}}{m_{e}}\right)^{2} \approx 3 \cdot 10^{9}
$$

(19) net gain of $\mathcal{O}\left(10^{3}\right)$

(12) drawback: lower energy available in the CMS of the vector bosons, because of soft bremsstrahlung spectrum (see below)

- upper Higgs mass reach of linear collider dominated by Vector Boson Fusion:

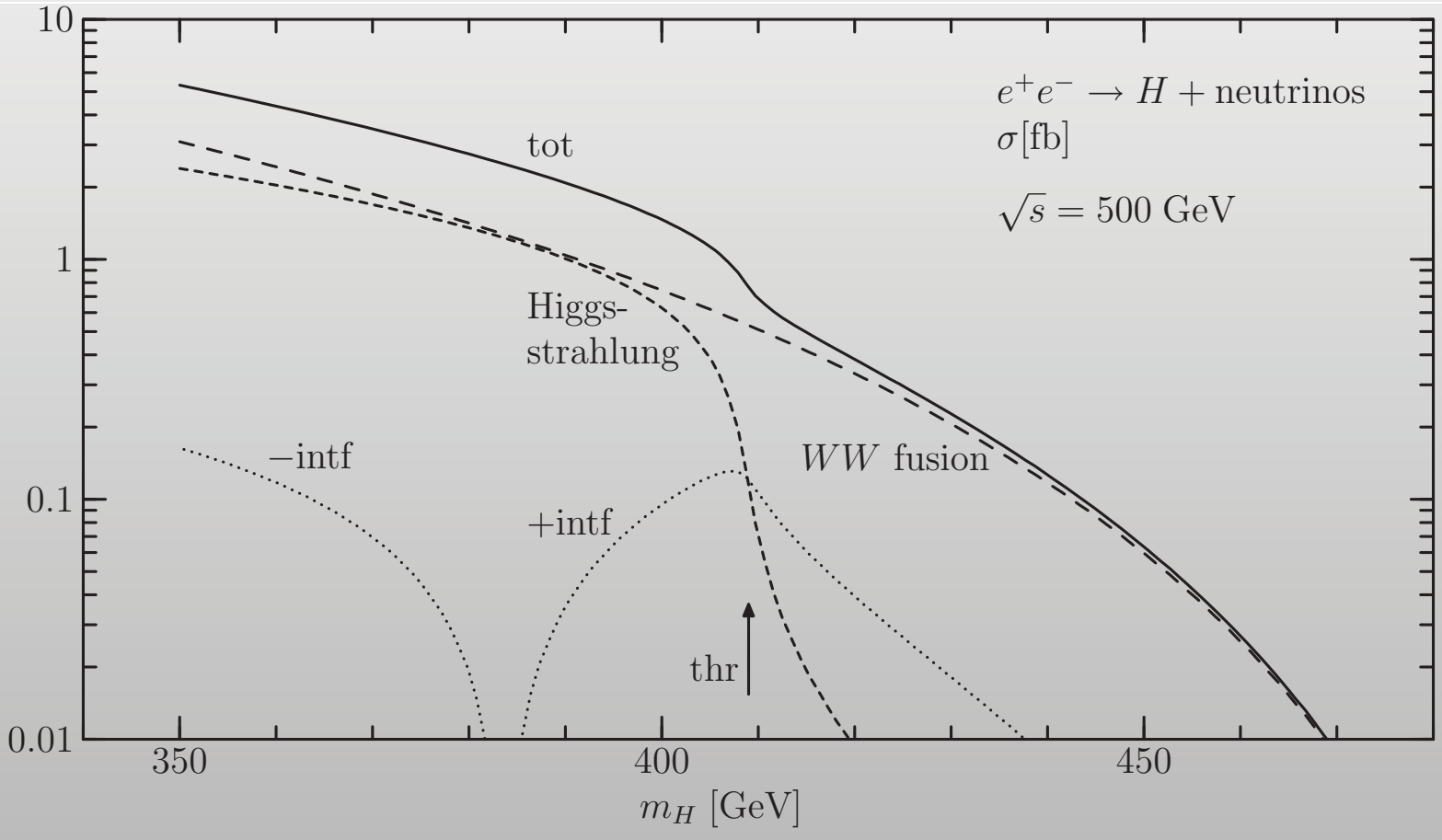


- 1990s: LEP enforced triumph of the minimal standard model

- 2000s: theorists running wild due to lack of supervision from experimentalists: plethora of new and repackaged BSM models

- 2010s: LHC

- will most of the content of arXiv. org be obsolete soon, or

- will we have to come up with completely new ideas?

- 2020s: ILC/CLIC: Will the fog be lifted? 